\title{
转甜菜碱醛脱氢酶基因烟草叶片中 抗氧化酶活性增高
}

骆爱玲 ${ }^{(1)}$ 刘家尧(1) 马德钦 ${ }^{(2)}$ 王学臣 ${ }^{(3)}$ 梁 峥 ${ }^{(1)}$

(1)中国科学院植物研究所, 北京 100093; (2)中国科学院微生物研究所, 北京 100080; (3)中国农业大学生物学院,

北京 100094. Email: liangzh@ns.ibcas.ac.cn)

摘要 转甜菜碱醛脱氢酶(BADH)基因烟草叶片中超氧物歧化酶(SOD)的活性比对照增加约 36\%, 过氧化氢酶(Cat)和过氧化物酶(POD)活性分别提高约 $62 \%$ 和 $88 \%$, 定位于叶绿体中的抗坏血酸谷胱甘肽途径的抗坏血酸过氧化物酶(AsAPOD), 脱氢抗坏血酸还原酶(DAsAR)和谷胱甘肽还原 酶(GR)活性分别提高 $67.7 \%, 47.9 \%$ 和 $38.8 \%$, 表明由 SOD 催化阴离子自由基 $\mathrm{O}_{2}^{-}$所产生的活性氧 $\mathrm{H}_{2} \mathrm{O}_{2}$ 能被完全分解, 不会衍生毒性很强的羟自由基 - $\mathrm{OH}$. 定量说明两类抗氧化酶分解自由基与 活性氧的相互匹配. 转基因植株的耐光抑制能力比对照提高幅度较大, 这可能是由于抗氧化酶活 性提高, 更有效清除了活性氧和自由基, 保护了叶绿体的结构与功能. 证明抗氧化酶活性的提高 可能是转 BADH 基因植株抗性提高的重要原因之一.

关键词 甜菜碱醛脱氢酶基因 烟草 抗氧化酶活性

植物线粒体和叶绿体的电子传递链发生电子漏(electron leakage)时, 电子传递给 $\mathrm{O}_{2}$ 产生 超氧阴离子自由基 $\mathrm{O}_{2}^{-}$, 后者由超氧物歧化酶(SOD)催化, 反应生成 $\mathrm{H}_{2} \mathrm{O}_{2}$ 和 $\mathrm{O}_{2}$ :

$$
2 \mathrm{O}_{2}^{-}+2 \mathrm{H}^{+} \stackrel{\mathrm{SOD}}{\longrightarrow} \mathrm{H}_{2} \mathrm{O}_{2}+\mathrm{O}_{2}
$$

$\mathrm{H}_{2} \mathrm{O}_{2}$ 再通过 Haber-Weiss 反应

$$
\mathrm{H}_{2} \mathrm{O}_{2}+\mathrm{O}_{2}^{-} \stackrel{\mathrm{Fe}^{3+}, \mathrm{Fe}^{2+}}{\longrightarrow} \mathrm{OH}^{-}+\mathrm{O}_{2}+\cdot \mathrm{OH}
$$

生成毒性最强的羟自由基 $\cdot \mathrm{OH}$.

环境胁迫对植物造成的伤害被认为是自由基所引起 ${ }^{[1]}$, 因而细胞中清除活性氧的能力常 被用来作为植物抗逆能力的生理指标. 细胞中要维持自由基代谢的平衡而不产生积累, 除需 有足够 SOD 活性及时转化 $\mathrm{O}_{2}^{-}$外, 还需要相匹配分解 $\mathrm{H}_{2} \mathrm{O}_{2}$ 的酶. 高等植物中存在两套分解 $\mathrm{H}_{2} \mathrm{O}_{2}$ 的酶系统, 一是细胞质中的过氧化氢酶 (Cat)和过氧化物酶(POD), 二是定位于叶绿体的 抗坏血酸-谷胱甘肽途径, 包括抗坏血酸过氧化物酶(AsAPOD)、脱氢抗坏血酸还原酶(DAsAR) 和谷胱甘肽还原酶(GR).

高等植物适应环境胁迫的重要生理对策之一是渗透调节, 为此细胞中积累大量有机渗调 物质, 甜菜碱就是最重要的渗调剂之一. 在高等植物中, 甜菜碱醛脱氢酶(BADH)是合成甜菜 碱的关键酶. 据报道, 转 BADH 基因的植物比较明显地提高了抗性 ${ }^{[2 \sim 4]}$. 这种抗性的提高最直 接的原因可能是由于增加了甜菜碱的合成, 同时也可能是改善了其他生理过程, 但这方面几 乎没有资料报道. 本文报道外源引入 $\mathrm{BADH}$ 基因后，烟草抗氧化酶活性的增加.

\section{1 材料与方法}

(i) 试验材料. 转 BADH 基因烟草(Nicotinan tabacum)是我们培育的 $\mathrm{K}_{326}$ 烟草 $\mathrm{T}_{2}$ 株系, 以亲本烟草植株为对照. 植株栽培方法如文献[5]. 株高约 $25 \mathrm{~cm}$ 时, 从上部取成熟全展开叶(通 
常为第 3 或第 4 片叶)测定抗氧化酶活性. 植株耐光抑制能力在株高约 $65 \mathrm{~cm}$ 时取叶片进行测定.

(ii) 酶活性测定. 取 $1 \mathrm{~g}$ 叶片加入 $2 \mathrm{~mL}$ 研磨介质在冰浴中充分研磨后, 经一层尼龙纱 布过滤，滤液于 $12000 \times g$ 离心 $15 \mathrm{~min}$, 上清液作酶分析用. 研磨介质的组成为: $50 \mathrm{mmol} / \mathrm{L}$ Tris- $\mathrm{HCl}(\mathrm{pH}=7.8), 0.2 \mathrm{mmol} / \mathrm{L}$ EDTA- $\mathrm{Na}_{2}, 10 \mathrm{mmol} / \mathrm{L} \beta$-㧧基乙醇, $0.2 \mathrm{~mol} / \mathrm{L}$ 蔗糖. 抗氧化酶 中的抗坏血酸氧化酶、脱氢抗坏血酸还原酶、谷胱甘肽还原酶和过氧化物酶活性参考 Cakmak 等人 ${ }^{[6]}$ 的方法测定, 过氧化氢酶活性测定按 Liang 等人 ${ }^{[7]}$ 的方法, 超氧物歧化酶活性测定按 Giannopolitis 等人 ${ }^{[8]}$ 的方法, 但光照强度为 $63 \mu \mathrm{E} \cdot \mathrm{s}^{-1} \cdot \mathrm{m}^{2}$, 测定 $560 \mathrm{~mm}$ 光密度变化, 以每 分钟引起抑制 NBT 光还原 0.01 的 $A$ 值计为 $1 \mathrm{U}$.

(iii) 叶片耐光抑制的测定. 从两株转基因烟草植株上部各取两片完全展开的成熟叶片, 按 $\mathrm{Jiao}^{[9]}$ 的方法测定叶片耐光抑制特性. 以亲本植株叶片为对照. 叶片置室外自然光下, $53 \mathrm{~h}$ 后观察叶片发黄情况. 用称重法计算发黄叶片的面积.

\section{2 结果}

\subsection{SOD, POD, Cat 活性的变化}

由表 1 可以看到, 对照 SOD 活性为 $11 \mathrm{U}$ 左右, 转基因烟草叶片的 SOD 活性约 $15 \mathrm{U}$, 即 提高 $36 \%$ 左右. 而对照 POD 和 Cat 活性分别为 1.16 和 $34.44 \mathrm{nmol} \cdot \mathrm{mg}^{-1}$ 蛋白 $\cdot \mathrm{min}^{-1}$, 转基因 植株为 1.88 和 $64.72 \mathrm{nmol} \cdot \mathrm{mg}^{-1}$ 蛋白 $\cdot \mathrm{min}^{-1}$, 即转基因烟草提高约 $60 \%$ 和 $90 \%$.

表 1 转基因烟草叶片中 SOD, POD 和 Cat 活性的变化

\begin{tabular}{|c|c|c|c|c|c|c|}
\hline \multirow{2}{*}{ 植物 } & \multicolumn{2}{|l|}{ SOD } & \multicolumn{2}{|l|}{ POD } & \multicolumn{2}{|l|}{ Cat } \\
\hline & $/ \mathrm{U} \cdot \mathrm{mg}^{-1}$ 蛋白 $\cdot \mathrm{min}^{-1}$ & $1 \%$ & $/ \mathrm{nmol} \cdot \mathrm{mg}^{-1}$ 蛋白 $\cdot \mathrm{min}^{-1}$ & $1 \%$ & $/ \mathrm{nmol} \cdot \mathrm{mg}^{-1}$ 蛋白 $\cdot \mathrm{min}^{-1}$ & $1 \%$ \\
\hline 转基因烟草 & 14.89 & 136.48 & 1.88 & 162.07 & 64.72 & 187.92 \\
\hline 对照 & 10.91 & 100 & 1.16 & 100 & 34.44 & 100 \\
\hline
\end{tabular}

2.2 抗坏血酸-谷胱甘肽途径中的抗氧化酶活性变化

表 2 结果表明, 转基因烟草叶片中抗坏血酸-谷胱甘肽途径中的 3 个抗氧化酶 AsAPOD, DAsAR 和 GR 的活性分别由对照 $20.68,39.58$ 和 $115.66 \mathrm{nmol} \cdot \mathrm{mg}^{-1}$ 蛋白 $\cdot \mathrm{min}^{-1}$ 增加到 34.68 , 58.55 和 $160.48 \mathrm{nmol} \cdot \mathrm{mg}^{-1}$ 蛋白 $\cdot \mathrm{min}^{-1}$, 即分别提高 $40 \%$ 70\%左右.

表 2 转基因植株中抗坏血酸-谷胱甘肽途径中的酶活性

\begin{tabular}{|c|c|c|c|c|c|c|}
\hline \multirow{2}{*}{ 植物 } & \multicolumn{2}{|l|}{ AsAPOD } & \multicolumn{2}{|l|}{ DAsAR } & \multicolumn{2}{|l|}{ GR } \\
\hline & $/ \mathrm{nmol} \cdot \mathrm{mg}^{-1}$ 蛋白 $\cdot \mathrm{min}^{-1}$ & $1 \%$ & $/ \mathrm{nmol} \cdot \mathrm{mg}^{-1}$ 蛋白 $\cdot \mathrm{min}^{-1}$ & $1 \%$ & $\mathrm{lnmol} \cdot \mathrm{mg}^{-1}$ 蛋白 $\cdot \mathrm{min}^{-1}$ & $1 \%$ \\
\hline 转基因植株 & 34.68 & 167.70 & 58.55 & 147.93 & 160.48 & 138.75 \\
\hline 对照 & 20.68 & 100 & 39.58 & 100 & 115.66 & 100 \\
\hline
\end{tabular}

\section{3 转基因烟草叶片耐光抑制能力的提高}

叶绿体中的分子氧的光还原是不可避免的, 并导致产生超氧阴离子自由基 $\mathrm{O}_{2}^{-}$和活性氧 $\mathrm{H}_{2} \mathrm{O}_{2}$, 这些活性氧和它们的衍生物羟自由基 $\cdot \mathrm{OH}$ 和单线态氧 ${ }^{1} \mathrm{O}_{2}$ 破坏叶绿体的完整性和功能. 叶绿体中存在抗坏血酸-谷胱甘肽途径和 $\mathrm{SOD}$, 可以消除活性氧 $\mathrm{H}_{2} \mathrm{O}_{2}$ 和超氧阴离子自由基 $\mathrm{O}_{2}^{-}$, 防止或减少这些活性氧的伤害. 在转 BADH 基因的烟草, 叶片中的各种抗氧化酶活性均有明 显提高(表 1 和 2), 暗示这些植株的抗光抑制能力会有明显增强. 
为了验证上述设想, 从两株转基因植株各取两片成熟全展叶片, 以亲本植株为对照, 进 行了叶片抗光抑制能力测定, 结果(表 3)表明, 转基因植株叶片的耐光抑制能力明显提高, 转 黄速度延缓. 对照叶片转黄速度加快, 转黄面积明显增加, 比转基因植株的面积多 1 倍, 而转 基因植株绿色叶面积比对照高 1 倍.

表 3 烟草叶片抗光抑制的能力(自然光照 $53 \mathrm{~h}$ )

\begin{tabular}{|c|c|c|c|c|c|c|}
\hline \multirow{2}{*}{ 植株 } & \multicolumn{2}{|c|}{ 叶片总面积 } & \multicolumn{2}{|c|}{ 黄叶面积 } & \multicolumn{2}{|c|}{ 绿叶面积 } \\
\hline & $/ \mathrm{cm}^{2}$ & $1 \%$ & $/ \mathrm{cm}^{2}$ & $1 \%$ & $/ \mathrm{cm}^{2}$ & $1 \%$ \\
\hline 转基因烟草 & 337.97 & 100 & 127.51 & 37.73 & 210.46 & 62.27 \\
\hline 对照 & 340.12 & 100 & 249.12 & 73.24 & 91.00 & 26.76 \\
\hline
\end{tabular}

\section{3 讨论}

关于自由基和活性氧与植物环境胁迫的关系, 由于 SOD 活性的单位与分解 $\mathrm{H}_{2} \mathrm{O}_{2}$ 酶活性 的单位不同, 在比较转化 $\mathrm{O}_{2}^{-}$的 SOD 的活性与分解 $\mathrm{H}_{2} \mathrm{O}_{2}$ 的酶活性是否平衡时是困难的, 所以 本文中以各抗氧化酶活性变化的百分数进行比较.

在植物体中, SOD 主要定位于叶绿体, 一部分存在于线粒体; 抗坏血酸-谷胱甘肽途径存 在于叶绿体; Cat 定位于过氧化物酶体; POD 存在于细胞溶质. 它们都是非膜结合的蛋白质, 在 提取这些酶时, 用的是低渗缓冲液(含 $0.2 \mathrm{~mol} / \mathrm{L}$ 蔗糖), 并经剧烈研磨, 在这种条件下, 各种细 胞器都破碎了 ${ }^{[7]}$, 所以所测酶活性可代表其所在细胞器中的活性. 细胞中的 $\mathrm{O}_{2}^{-}$和 $\mathrm{H}_{2} \mathrm{O}_{2}$ 是很小 的分子, 可自由透过细胞器膜与酶反应. 假定细胞中分解超氧物自由基按反应(1)进行, 消除 活性氧 $\mathrm{H}_{2} \mathrm{O}_{2}$ 的两套酶系统在体内正常运转, 在转基因植株中 SOD 活性提高 36\%, 而 POD 和 Cat 的活性分别约为 SOD 的 2 倍, 总计提高 3 倍左右, 也就是说, 由 SOD 转化 $\mathrm{O}_{2}^{-}$时所产生 的 $\mathrm{H}_{2} \mathrm{O}_{2}$ 是能够完全被 POD 和 Cat 消除的. 在叶绿体, $\mathrm{H}_{2} \mathrm{O}_{2}$ 由抗坏血酸-谷胱甘肽途径消除, 从 组成途径的 3 个酶活性变化看, 其限制因素是 GR 活性(表 2), 在转基因植株其活性提高 38\%, 也基本能够满足清除由于 SOD 活性提高(表 1,36\%)而多产生的 $\mathrm{H}_{2} \mathrm{O}_{2}$. 表 1 和 2 的结果表明, 转 基因植株清除 $\mathrm{H}_{2} \mathrm{O}_{2}$ 酶活性提高总计约为 SOD 的 4 倍, 对于清除由反应(1)产生的 $\mathrm{H}_{2} \mathrm{O}_{2}$ 是足够 的. SOD 活性的提高与分解 $\mathrm{H}_{2} \mathrm{O}_{2}$ 酶活性提高是相匹配的.

在植物细胞中, 除了由光合电子传递链和细胞呼吸链的电子漏形成的 $\mathrm{O}_{2}^{-}$而产生 $\mathrm{H}_{2} \mathrm{O}_{2}$ 外, 还有两条产生 $\mathrm{H}_{2} \mathrm{O}_{2}$ 的途径, 即定位于过氧化物酶体的光呼吸和主要定位于乙醛酸循环体的脂 肪酸的 $\beta$-氧化. 由于转基因烟草中分解 $\mathrm{H}_{2} \mathrm{O}_{2}$ 的酶活性的提高比 SOD 的活性提高多 3 倍, 可 以相当大程度上清除来自光呼吸和脂肪酸 $\beta$-氧化产生的 $\mathrm{H}_{2} \mathrm{O}_{2}$. 可能正是由于抗氧化酶活性的 提高, 从而增加了转基因植株耐光抑制活性. 在叶绿体清除自由基和活性氧的限制因素是 SOD 和 GR(表 1 和 2), 在转基因植株中两者提高约 36\%. 转基因植株在受光胁迫后, 黄叶面 积减少而绿叶面积相应增加 $35 \%$ 左右, 说明耐光抑制的增加可能正是由于抗氧化酶活性提高 的结果. 在植物细胞中, BADH 被认为定位在叶绿体 ${ }^{[10]}$, 最近的研究表明, 在过氧化物酶体也 有存在 ${ }^{[11,12]}$, 将外源 BADH 基因导入烟草, BADH 基因定位在叶绿体, 叶片细胞可活跃地将外 源甜菜碱醛转变为甜菜碱, 其含量可达 $20 \mu \mathrm{mol} / \mathrm{g}$ 鲜重, 比对照明显增加 ${ }^{[13]}$. 甜菜碱对光合放 氧中心的结构和功能 ${ }^{[14,15]}$ 和各种呼吸酶 ${ }^{[16]}$ 有很好的保护作用. 转基因植株各种抗氧化酶活性 增加是否由于甜菜碱的增加还不清楚, 甜菜碱对各种抗氧化酶的结构与功能的影响未见报道. 
但本文的结果为我们以前提出过的 ${ }^{[5]}$ 转 $\mathrm{BADH}$ 基因烟草可能增加耐光抑制能力提供了证据, 与我们的观点一BADH 基因的导入可能增加植株重要的“非渗透调节”功能, 以及外源基因

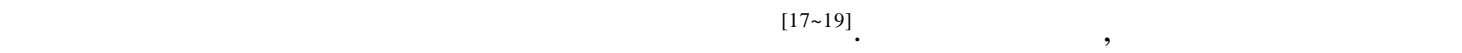
可能也是转 BADH 基因植物增加抗逆性的重要原因之一. Nuccio 等人 ${ }^{[20]}$ 和 Smirnoff ${ }^{[21]}$ 最近指 出, 外源导入工程途径可能产生不合需要的副效应( side effects), 抗渗透胁迫代谢工程下一步 的重点是大量增加渗调保护剂的同时不损伤其他代谢过程. 这些看法与本文中将 BADH 基因 导入烟草引起其抗氧化酶活性提高和抗光抑制能力的增高是相一致的.

致谢 本工作为国家基础研究发展规划(G1999011700)、国家 “攀登” 计划和山东省自然科学基金(批 准号: Y98D23071)资助项目.

\section{参考文献}

1 王爱国. 植物的氧代谢. 见: 余叔文, 汤章城, 主编. 植物生理与分子生物学. 第 2 版. 北京: 科学出版社, 1998. 366 389

2 梁 峥, 马德钦, 汤 岗, 等. 菠菜甜菜碱醛脱氢酶基因在烟草中的表达. 生物工程学报, 1997, 13(3): 236 240

3 刘风华, 郭 岩, 谷冬梅, 等. 转甜菜碱醛脱氢酶基因植物耐盐性的研究. 遗传学报, 1997, 24: 54 58

4 Hayashi H, Murata N. Genetically engineered enhancement of salt tolerance in higher plants. In: Satoh K, Murata N, eds. Stress Responses of Photosynthetic Organisms. New York: Elsevier, 1998. 133 148

5 衣艳君, 刘家尧, 骆爱玲, 等. 转 BADH 基因烟草的光系统 II 和呼吸酶活性变化. 植物学报, 1999, 41: 993 996

6 Cakmak J, Marschner H. Magnesium deficiency and high light intensity enhance activities of superoxide dismutase, ascorbate peroxidase and glutathione reductase in bean leaves. Plant Physiol, 1992, 98(4): 1222 1227

7 Liang Z, Yu C, Huang A H C. Isolation of spinach leaf peroxisomes in 0.25 molar sucrose solution by percoll density gradient centrifugation. Plant Physiol, 1982, 70(4): 1210 1222

Giannopolitis C N, Ries S K. Superoxide dismutase I , occurrence in higher plants. Plant Physiol, 1977, 59(2): 309 314

9 Jiao D M. Mass screening for rice germplasm tolerant to photoinhibition. Photosynthtica, 1992, 26(3): 397 404

10 Hanson A D, May A M, Grumet R, et al. Betaine synthese in chenopodes: localization in chloroplasts. Proc Natl Acad Sci USA, 1985, 82(11): 3678 3682

11 Nakamura T, Yokota S, Muramoto Y, et al. Expression of a betaine aldehyde dehydrogenase gene in rice, a glycinebetaine nonaccmulator and possible localization of its protein in peroxisomes. Plant J, 1997, 11(5): 1115 1120

骆爱玲, 赵 原, 李浴春, 等. 甜菜碱醛脱氢酶在菠菜叶细胞中的定位. 植物生理学报, 1995, 21(2): 117 122

Rathinasabapathi B, McCue K F, Gage D A, et al. Metabolic engineering of glycine betaine synthesis: plant betaine aldehyde dehydrogenase lacking typical transit peptides are targeted to tobacco chloroplasts where they confer betaine aldehyde resistance. Planta, 1994, 193(2): 155 162

侯彩霞, 徐春和, 汤章城, 等. 甜菜碱对 PS II 放氧中心结构的选择性保护. 科学通报, 1997, 42(17): 1857 1859

Papageorgiou G C, Murata N. The unusually strong stabilizing effects of glycine betaine on the structure and function of the oxygen-evolving photosystem II complex. Photosynthesis Research, 1995, 44(3): 243 252

梁 峥, 赵 原, 汤 岗, 等. 甜菜碱对呼吸酶的保护效应. 植物学报, 1994, 36(12): 945 947

汤佩松. 高等植物呼吸代谢途径的调节控制和代谢与生理功能间的相互制约. 植物学报, 1979, 21(2): 93 106

梁 峥, 梁厚果. 植物呼吸代谢. 见: 余叔文, 汤章城, 主编. 植物生理与分子生物学. 第 2 版, 北京: 科学出版社, 1998. 344 365

19 刘家尧, 王学臣, 梁 峥. 植物基因表达的代谢调控. 植物学通报, 1999, 16(1): 1 10

20 Nuccio M L, Rhodes D, Mcneil S D, et al. Metabolic engineering of plants for osmotic stress resistance. Current Opinion in Plant Biology, 1999, 2(2): 128 134

21 Smirnoff N. Plant resistance to environmental stress. Current Opinion in Biotechnology, 1998, 9(2): 214 219 